\title{
BMJ Open Enhancing intrinsic motivation for physical activity among adolescents with cystic fibrosis: a qualitative study of the views of healthcare professionals
}

\author{
Sarah Denford, ${ }^{1}$ Kelly A Mackintosh, ${ }^{2}$ Melitta A McNarry, ${ }^{2}$ Alan R Barker, ${ }^{1}$ \\ Craig Anthony Williams, ${ }^{1}$ On behalf of Youth Activity Unlimited - A Strategic \\ Research Centre of the UK Cystic Fibrosis Trust
}

To cite: Denford S,

Mackintosh KA, McNarry MA, et al. Enhancing intrinsic motivation for physical activity among adolescents with cystic fibrosis: a qualitative study of the views of healthcare professionals. BMJ Open 2019;9:e028996. doi:10.1136/ bmjopen-2019-028996

- Prepublication history for this paper is available online. To view these files, please visit the journal online (http://dx.doi. org/10.1136/bmjopen-2019028996).

Received 14 January 2019 Revised 30 April 2019 Accepted 9 May 2019

Check for updates

(c) Author(s) (or their employer(s)) 2019. Re-use permitted under CC BY-NC. No commercial re-use. See rights and permissions. Published by BMJ.

${ }^{1}$ Children's Health \& Exercise Research Centre, Sport and Health Sciences, University of Exeter, Exeter, UK

${ }^{2}$ Applied Sports Science, Technology, Exercise and Medicine Research Centre, College of Engineering, Swansea University, Swansea, UK

Correspondence to

Dr Sarah Denford;

s.denford@exeter.ac.uk

\section{ABSTRACT}

Objective To explore the views of healthcare professionals from cystic fibrosis (CF) multidisciplinary teams (MDT) on physical activity for adolescents with CF, the specific strategies used for physical activity promotion and associated challenges.

Design In this exploratory study, in-depth qualitative interviews were conducted with 15 healthcare professionals from CF MDTs to explore their views surrounding physical activity promotion for adolescents with CF.

Participants Eleven physiotherapists (nine female), two consultants (both male) and two dieticians (both female) provided written informed consent and participated in the study.

Setting CF clinics in the UK.

Results While healthcare professionals highlighted the importance of physical activity in the management of CF, they noted that very few patients were motivated solely by (CF or general) health reasons. Healthcare professionals discussed the need for physical activity to be an enjoyable and routine part of their life, undertaken with significant others, outside the clinic whenever possible. Adopted approaches for physical activity promotion focused on providing individualised recommendations that suit the patients' individual needs and goals and enhance intrinsic motivation for physical activity.

Conclusion Our research offers valuable information for those seeking to develop interventions to promote physical activity among adolescents with CF. Specifically, intervention developers should focus on developing individualised interventions that focus on enhancing intrinsic motivation and support the integration of physical activity into everyday life.

\section{INTRODUCTION}

Cystic fibrosis (CF) is the most common life-limiting genetic condition in the UK, affecting more than 10400 people. ${ }^{1}$ Due to advances in treatment, screening and infection control, people with CF have a greater life expectancy than in previous years, ${ }^{2}$ with the $\mathrm{UK}$ CF registry stating that the average
Strengths and limitations of this study

- Qualitative methods generated an in-depth account of the views and practices of an understudied group of participants.

- Multiple coders, respondent validation and triangulation of findings with the existing literature enhances the trustworthiness of our data.

- Convenience sampling may have resulted in a biased sample of participants.

- Further work is needed to explore the perspectives of young people with cystic fibrosis.

life expectancy of individuals with $\mathrm{CF}$ in the UK is now 47 years ${ }^{3}$. However, treatment is demanding, ${ }^{4}$ and involves a complex combination of symptomatic and prophylactic daily medications, physiotherapy and airway clearance, high-calorie diets and antibiotic therapy in the event of respiratory infection. ${ }^{5}$

Physical activity and exercise programmes, now embedded into standards of care, are considered to be positive and important aspects of treatment. ${ }^{6}$ Systematic reviews show that physical exercise training can improve aerobic capacity, lung function and health-related quality of life. ${ }^{7}$ Furthermore, qualitative studies report that adolescents with CF benefit greatly from physical activity and exercise, and not just in terms of physiological benefits. Specifically, the literature includes examples of people with CF reporting considerable feelings of accomplishment after significant physical challenges, ${ }^{8}$ positive affect as a result of physical activity, ${ }^{9}{ }^{10}$ an increased sense of empowerment over their condition ${ }^{9}$ and increased opportunities for recreational activities with their peers. ${ }^{11}$ Moreover, converse to other clinical populations, some studies have reported that some youth with CF may have similar or higher levels of physical 
activity than their peers, both with and without chronic conditions. $^{12-14}$

However, irrespective of condition, there is a notable decline in physical activity during adolescence, with girls showing the greatest decline. ${ }^{12}$ Reductions in physical activity and exercise during this period can track into adulthood, and may therefore have serious long-term implications. ${ }^{15-17}$ Specifically, declines in physical activity may be associated with limiting individuals' social opportunities (eg, not being able to take part in physically active events with peers) and a decline in pulmonary function, potentially leading to a cycle of deconditioning and reduction in aerobic fitness, which is known to be an important predictor of survival. For physical activity to be integrated into the lives of young people with $\mathrm{CF}$, it has to be something that they are motivated to do.

Self-determination theory ${ }^{18}$ is often used to explain, predict or change physical activity behaviour among both healthy ${ }^{19}$ and clinical populations. ${ }^{20}$ The theory describes two main types of motivation: intrinsic and extrinsic motivation. Individuals who are intrinsically motivated to be active do so purely for the pleasure of being active. Motivation for the behaviour comes from within the individual and is the most autonomous form of motivation. Because motivation for the behaviour is not dependent on external forces, it is likely to be sustained-even when circumstances change. However, many individuals are active only because of what the self-determination theory refers to as controlled motivation. This includes external regulation, in which a person acts to gain reward or avoid punishment, and introjected regulation, in which people are active to avoid feelings of guilt or shame, or to increase self-esteem or pride. Healthcare professionals often create controlled motivation through offering incentives for physical activity or by creating feelings of guilt if physical activity is avoided. While this may lead to short-term increases in physical activity, this is unlikely to be sustained over time.

It is argued that autonomy supportive environments, in which the individual is given information and encouragement rather than instructions and choices are respected, will foster autonomous intrinsic motivation. Motivational interviewing ${ }^{21}$ is a set of behaviour change techniques (BCTs), widely used in interventions based on the self-determination theory ${ }^{22}{ }^{23}$ to enhance autonomy and promote individuals taking responsibility for their behaviour. Indeed, there is strong evidence for the effectiveness of interventions using motivational interviewing to promote physical activity among clinical populations. ${ }^{24}$

The need to consider the perspective of all stakeholders when developing interventions is widely acknowledged..$^{25}$ As healthcare professionals are tasked with increasing physical activity levels among people with $\mathrm{CF}^{1}$ and promote physical activity to a wide range of individuals on a daily basis, they are ideally placed to contribute to the development of interventions to support the promotion of physical activity for young people with CF. Previous research has found that physical activity as a treatment is highly valued by CF teams who recognise its therapeutic impact and potential to improve patients' health. ${ }^{26}$ However, although healthcare providers report discussing adherence at every opportunity, and frequently use strategies to increase knowledge about adherence to treatment, ${ }^{27}$ it is unclear how healthcare professionals attempt to increase motivation for physical activity among young people with CF. Gaining an understanding of current opinions and practice is crucial if researchers are to develop effective strategies to support clinicians prescribing physical activity in the future. ${ }^{25}$ Therefore, the purpose of the current study is to identify the views of multidisciplinary teams (MDTs) on physical activity for adolescents with CF, the specific strategies used for physical activity promotion and associated challenges. This information has the potential to inform the development of educational materials to better support MDTs to promote and implement physical activity for adolescents with $\mathrm{CF}$.

\section{METHODS \\ Design}

In this exploratory study, in-depth qualitative interviews were held with healthcare professionals from CF MDTs to explore their views surrounding physical activity promotion for adolescents with CF. This work was conducted in accordance with the Standards for Reporting Qualitative Research .

\section{Participant sampling and data collection}

Convenience sampling was used to recruit practitioners in the UK. Information about the study was distributed via email to all physiotherapists, paediatricians, dieticians and nurses who are currently on the Cystic Fibrosis Trust distribution list. Eleven physiotherapists (nine female), two consultants (both male) and two dieticians (both female) provided written informed consent and participated in the study. Participants were recruited from eight clinics in the South East, South West and North West England and two clinics in Scotland.

Participants were invited to take part in telephone interviews in which open-ended questions were used to explore healthcare professionals' views and opinions about promoting physical activity for adolescents with $\mathrm{CF}$, the strategies they use and any barriers they perceived. The initial interview schedule (online supplementary 1) was based on a review of the literature and consultations with CF practitioners, including a paediatrician and a physiotherapist from our network. Participants were informed that interviews would last between 20 and $40 \mathrm{~min}$ and asked to identify a suitable time for the interview to take place. Interviews ranged in duration from 24 to $42 \mathrm{~min}$, with the mean duration being $36 \mathrm{~min}$. While participants were asked specifically about their attempts to promote physical activity, many spontaneously referred to exercise, or referred to exercise and physical activity interchangeably. 


\section{Data analysis}

Interviews were audiorecorded and transcribed verbatim. Data were inductively analysed using a thematic approach. ${ }^{28}$ To enhance rigour, two researchers (SD and KAM) independently read and coded the transcripts to produce a list of core codes. A preliminary list of themes were developed and refined. ${ }^{29}$ In line with the six stages of thematic analysis, ${ }^{20}$ a chart was developed for each theme, ${ }^{29}$ and relevant data were entered into each chart. Charts were then used to identify narratives within cases and diversity between cases. ${ }^{30}$

Data were stored in, and analysed using, Nvivo 12. To ensure sensitivity to context, ${ }^{31}$ potential links to existing theories and previous research were sought and noted. Cases or themes that did not fit the developing analysis framework were actively sought and explanations discussed. To promote transparency, a record of the development of new codes, themes and patterns were kept in the form of a reflective analysis diary. ${ }^{31}$ To enhance validity, emergent ideas and initial interpretations of the data were discussed with a third author (MAM) who triangulated the data in reverse, from the themes back to the original transcripts, challenging interpretations until a consensus was reached. Furthermore, a summary of the findings were sent to all participants along with an invitation to offer any comments. Four healthcare professionals responded and were in agreement with our interpretation of the data.

\section{Patient and public involvement}

A patient and public involvement group was established to inform the development and direction of our research. The group met regularly (via skype) and comprised six individuals with CF, two parents, two physiotherapists, one technician and one paediatrician. This group were distinct from our research participants, and their main role was to inform the direction of the research that we conduct. In the first instance, the group were asked to suggest research topics and questions relating to physical activity and CF that they would like to be answered. The group were later asked to comment on our proposed methodology, information and materials for participants and semi-structured interview schedule.

\section{RESULTS}

The data are presented under two main headings: (1) drivers of physical activity behaviour and (2) changing physical activity behaviour. Pseudonyms and job titles are provided alongside each quote.

\section{Drivers of physical activity behaviour}

Healthcare professionals reported two main drivers of physical activity: health and enjoyment (table 1). While healthcare providers tend to prioritise improvements in markers of disease, such as lung function, this was rarely the main goal for the patients. Indeed, although

Table 1 Drivers of physical activity behaviour

"One chap who used to be a physical trainer, and he does say that the reason he enjoyed his exercise is that it

Quote 1 made him healthy and kept his numbers good... He's the only one though" (Victoria, physio)

Quote 2 "So even though we're drilling it into them that it's really important to exercise and it's going to keep you well, it's going to keep you better, the fitter you are the better you're going to be - if they're not interested they're not going to do it" (Corrine, physio).

Quote 3 "If they can't do it as well, it might be something they might measure their lung function against. So it, if they can't achieve, you know, he can't run as far now or as far as before he knows his function isn't as good and therefore..." (Beth, physio)

Quote 4 "If it's part of their treatment it seems to tail off, if they just do it for that. But if there is an element of enjoyment, one of ours, she enjoys running so she carries on - as opposed to seeing it as a treatment"

Quote 5 "I think [the biggest motivator] is just enjoyment. People get different physical feedback from exercise and people get really good endorphins and they want to be part of a team and they want to play as part of a team and want to socialise with friends in that way and some don't" (Shannon, dietician).

Quote 6 "I think the biggest barrier is the thing with any adolescents I think is you've got to motivate them they've got to want to do it and say if you're not interested doing it then that's really difficult" (Peter, physio).

Quote 7 "I think sometimes parents (are barriers to PA). If they don't exercise themselves, then the patients aren't enthusiastic about exercising because they don't have a positive role model" (James, paediatrician).

Quote 8 "I think some lads who aren't 'sporty' wouldn't necessarily join in because their families aren't going 'come on let's go for a walk or a bike ride'. And allow them from an early age to be on mobile devices" (Beth, physio).

Quote 9 "I think if the family is focused and the patient is focused on activities they can usually overcome any financial burden" (Alice, physio).

Quote 10 "When you get friendship groups doing it, I think that helps, so they're just joining what their friends are doing instead of going, you know, to some hospital team saying why don't you do exercise?" (Rachel, dietician).

Quote 11 "If their friends don't do activities then that's really difficult, that's the biggest thing" (Peter, physio).

Note: All names provided are pseudonyms. 
healthcare professionals suggested that improvements in health can be motivational, this was the exception rather than the rule (Quote 1). Generally, healthcare professionals suggested that for the majority of patients, motivation arising from the health benefits of physical activity is insufficient to increase physical activity behaviour (Quote 2). Furthermore, highlighting the correlation between lung function and physical activity could result in patients interpreting physical ability as a visible marker of lung function. For those who had poorer or declining health, this could be worrying for the patient and lead to an avoidance of physical activity (Quote 3).

Intrinsic motivation refers to both enjoyment and feelings of accomplishment from physical activity. Nearly all healthcare professionals emphasised the need for patients to enjoy physical activity. Crucially, it was consistently reported that those who enjoyed physical activity and felt good after physical activity were more motivated than those who felt it to be a chore (Quote 4). Indeed, feelings of enjoyment were considered to be the main factor that separated those who were active (Quote 5) from those who were not (Quote 6).

Nearly all healthcare professionals spoke of the importance of significant others in supporting patients to be active; usually through enhancing enjoyment. In younger patients, the role of the family was thought to be fundamental. During childhood, parents own exercise behaviour and their encouragement was considered the key predictor of activity behaviour (Quote 7). Families were seen to influence the patients' identity-either as sporty or not (Quote 8). However, family support and encouragement could help patients overcome possible barriers to being active if they were sufficiently engaged (Quote 9).

During adolescence, there is a shift from parent to peer support. Peer groups for adolescence with CF can act either as an enabler, or a deterrent. Healthcare professionals described the need for peers to support the person with $\mathrm{CF}$ to be active, as adolescents may prioritise fitting in and socialising with peers over optimal self-care. For some, friendship groups encouraged and promoted physical activity (Quote 10). However, for the adolescent, for whom fitting in and being accepted is paramount, inactive peer groups could reduce activity levels of the patient (Quote 11).

\section{CHANGING PHYSICAL ACTIVITY BEHAVIOUR}

Numerous BCTs for promoting physical activty behaviour were mentioned for overcoming the barriers highlighted by healthcare professionals. These primarily focused around (1) individualised education, (2) approaches used to enhance enjoyment and (3) approaches used to make physical activity a normal part of everyday life. The theme of providing an individualised approach and enhancing intrinsic motivation by making physical activity fun and enjoyable, sociable and normal ran throughout. Healthcare professionals spoke of creating a culture of exercise in which everyone is active. The need to form a united front, including all members of the team, the parents and the patients' friends was considered to be crucial. Particular behaviour change strategies are discussed below.

\section{Individualised education}

The most commonly mentioned BCT was individualised education about the benefits of physical activity, intensities and duration of physical activity and about how activity can be fitted into their lives (table 2). Education was usually verbally delivered by physiotherapists during consultations, or through recommendations of useful websites. Healthcare professionals were confident that their patients were well informed about the benefits of physical activity but would always reiterate the importance of physical activity at every session to highlight its importance. Despite the majority of healthcare professionals suggesting that patients are rarely motivated by health benefits, education about the benefits of physical activity was still a key part of consultations-as this was seen to be crucial to allow the patient to make an informed choice about their physical activity. The majority of education provided by healthcare professionals was individualised to the patients' individual needs, preferences and motivation (Quote 1). While healthcare professionals had 'ideal' or 'optimal' intensities and durations of physical activity in mind, there was an understanding that such advice would often be ignored and individualised recommendations are needed (Quote 2 and 3). Crucially, any recommendations would be patient-led and focus on individual situations, needs and goals (Quote 4 and 5). However, the resources (time and skill) for such an individualised approach were often lacking (Quote 6).

\section{Approaches to enhance enjoyment}

In line with healthcare professionals' belief that the biggest motivator is enjoyment, numerous attempts were made to make physical activity enjoyable to the patient: either by building intrinsic or extrinsic motivation (table 3). Attempts to increase enjoyment included attempting to identify types of physical activity that the patient would engage with (Quote 1). Healthcare professionals would make suggestions to encourage patients to try new things, or give patients opportunities to allow them to identify activities they enjoy (Quote 2 and 3). For some healthcare professionals, it could be absolutely anything that made patients move and laugh (Quote 4). However, again, suggestions would be tailored to each individual patient in accordance with their likes, dislikes and goals (Quote 5).

Importantly, it was noted that enjoyment would be more likely to lead to sustainable physical activity. 'Forcing' the patients to be active will never be effective in the longer term (Quote 6). Competitions were often used to enhance enjoyment, with some hospitals initiating leader boards, incorporating both staff and patients exercise test results (Quote 7) or developing challenges for their patients (Quote 8). However, it was noted that this would 
Table 2 Individualised education

"We talk to them about what they choose to do or not to do. It's a bit of repetition of information. Whether they choose to do anything with it or not is up to them. But I think that my sense is that if we're repeatedly giving that message, they realise the importance of it... So yeah, over time, hopefully they start to get the message that we

Quote 1 actually think it could help them and improve their lung function" (Louis, paediatrician).

Quote 2 "We do ask them every time we meet them what kind of exercise are you doing the intensity and the duration and really talk about it needs to be at half an hour at least and be high intensity" (Claire, physio).

Quote 3 "I think sometimes you just have to accept what they are willing to do, so it might not be optimal in terms of health, but it is a start. And it is better than not being able to do anything. It gives us something to work with. So it might not have the health benefits that we know comes with being active, but if they enjoy it, if they are still moving and being active and interacting with peers, then yes they are getting benefit from it. And it is a starting point." (Beth, physio).

\section{Quote 4 "I think a lot of it is very patient led rather than sort of saying this is a sort of set programme that we're working} towards" (Peter, physio).

Quote 5 "Yeah, it's very much an individualised thing, to fit in with what that person does or wants to do and what they want to achieve. We would talk about their goals and work with them to optimise their nutrition to achieve those goals. It wouldn't be a one size fits all, it would be a very individualised thing, taking into account things like current weight and fitness levels, what they want to achieve, whether they want to lose weight or whether it's just a fitness thing, whether they, what their diet currently looks like, all those sorts of things, and then we would work together to support that patient" (Rebecca, physio).

\section{Quote 6 "I think that we have to make individual exercise programmes for our patients depending on what their needs, their} social circumstances are and we just don't have the resources" (Peter, physio).

Note: All names provided are pseudonyms.

be less useful for people who are not already motivated (Quote 9).

Friends and family were often integral to attempts to make activity fun. For the younger patients, healthcare professionals would attempt to encourage parents to be active (Quote 10). For the older patients, peer groups were considered to be more influential, and healthcare professionals stated that they would often encourage patients to attend activity sessions with friends (Quote 11). If attempts at building intrinsic motivation were not effective, attempts were made to increase enjoyment via rewards. This could be as little as a reward at the end of a walk (Quote 12).

\section{Approaches used to make activity normal}

Healthcare professionals expressed the need to make activity integral to everyday life, rather than an additional treatment (table 4). Many healthcare professionals suggested that physical activity was crucial for all, not just those with a chronic condition. It was suggested that we need to develop a culture of exercise, in which everyone is active, and being active just becomes routine. In this instance, individuals with $\mathrm{CF}$ would not stand out or be different for their relationship with physical activity, rather, it would be standard practice for everyone. Techniques for 'making activity normal' centred around developing habits and routines, fitting physical activity into everyday life, outreach with schools and communities and promoting physical activity as a standard, rather than a treatment. Healthcare professionals attempted to encourage patients to develop habits and routines to enable physical activity to become part of everyday life. Early life experiences were considered to set a good foundation for viewing physical activity in a positive light (Quotes 1 and 2).

School and community involvement were considered potential avenues to support and promote physical activity-focusing on the generic health benefits of physical activity rather than illness prevention just for those with chronic conditions (Quote 3 and 4). It was acknowledged that physical activity should be for everyone and that everyone, irrespective of health condition, should be, and can benefit from being, active (Quote 5).

\section{DISCUSSION}

The present study interviewed 15 members of various CF MDTs in the UK in order to ascertain their views on physical activity promotion for adolescents with CF. The present study extended previous research, by discussing possible motivational influences that healthcare professionals believed to impact adolescents' physical activity levels, as well as presenting a range of BCTs intended to increase motivation. Within the current study, healthcare professionals highlight the need to provide an individualised approach to physical activity promotion, incorporating patients' needs, preferences and goals and focus on enhancing intrinsic motivation for physical activity by making it an enjoyable and routine part of their day. If supported by data obtained from other stakeholders (eg, young people with $\mathrm{CF}$ ), intervention developers could attempt to incorporate such factors into future interventions.

Of particular relevance to clinical teams is the suggestion that physical activity and exercise should be viewed as 
Table 3 Approaches used to enhance enjoyment

"I think it's finding something that connects with that person because there's no sort of bullet for, footballs not going to be for every boy or girl and gymnastics isn't going to be for everyone, dancing isn't going to be for

Quote 1 everyone" (Shannon, dietician).

Quote 2 'We will give them ideas, we will say 'Have you tried this?' And we say 'Is there any PE that you like?' And they might say trampolining, I really enjoy it, so we will say 'well why don't you think about doing it after school?' Or 'why don't you try that?' or 'why don't you try on a weekend?' And we'll try and use whatever they're doing in school or what their friends are up to. Or we'll say 'have you heard about that park run?'" (Rebecca, physio).

Quote 3 "You've got to give them a grass roots introduction to a lot of different options and opportunities and then find what they engage with and what they connect with most" (Claire, physio).

Quote 4 "I suggest to them to do absolutely anything that would get them off their bottoms. Because obviously my idea of an exercise opportunity and what I find fun is not necessarily what somebody else finds fun so I encourage them to find something that makes them laugh, makes them have fun and that they will continue to go back to" (Victoria, physio).

Quote 5 "I suppose the only thing is to spend time finding out what people like, so this is something we do, for example, so this allows us perhaps to target particular exercises and match particular people to, so we have a girl who likes to do Yoga so we devised a way of using the Yoga positions for drainage and using the breathing techniques to then encourage expansion in those particular positions. So I think the fact we're lucky in the small number that we have and we can get to know them well enough that we can kind of get deep, individual, personalised approach" (Claire, physio).

Quote 6 "We need to be more proactive with trying to not force our kids into exercise but to support them with getting to know what's out there and finding something that they can engage with and then it's not just the initiation of that programme it's the continuing support" (John, physio).

Quote 7 "We have a 'top gear' sort of thing where people that work in the hospital have done the bike test, so that the patients can think 'oh I want to try and beat a certain staff member' so they can see that all we're asking them to do, we're not all super fit either, and they can try and beat the next person the next time" (Jessica, physio).

Quote 8 "Before Christmas we did a challenge of ' $n$ ' kilometres. So we set a challenge with all the children messaging in how many kilometres that they've done a week. So we have a couple that will do a park run so they say well I've done 5K this week so we add that to our tally and we want to get a thousand kilometres between us" (Beth, physio).

Quote 9 "It's only good for the children that actually care and think 'Oh I need to get a couple more thousand steps in.' If not it's just a number on a watch" (Peter, physio).

Quote 10 "We ask parents whether they as a family do any exercise and look at trying to do exercises together as part of a family so it's not a physio thing to do, it's more a fun thing that they all do together" (Victoria, physio).

Quote 11 "We've tried in the past going out to see somebody at home and I would get her friend there as well so the two of them would be doing like a body pump style session in the house just because if she just wouldn't do it by herself but her friend was really keen to give it a go" (Claire, physio).

Quote 12 "With the slightly older ones, we might have a walk around the hospital, sometimes it might even be a bit of a walk into town, but with a bit more of an incentive of like going to like a milkshake shop or something with them just to encourage them to get out and have it's like a bit of a treat at the end of it" (Alice, physio).

Note: All names provided are pseudonyms.

'fun' rather than 'medicine'. Indeed, healthcare professionals were adamant that physical activity should be a pleasurable activity that the patient can enjoy with friends and/or family and not just an additional treatment. This raises significant questions regarding who is best placed to promote physical activity for clinical populations. In 2007, the American Medical Association and the American College of Sports Medicine launched the 'Exercise is Medicine' (EIM) initiative ${ }^{32}$ with the goal of increasing exercise assessment and promotion by clinical teams. Critically, healthcare professionals in our study suggest that this may not be the best approach for achieving longterm, sustainable behaviour change. They emphasised that physical activity and exercise should be promoted as a fun and enjoyable part of everyday life, because patients are unlikely to be motivated to exercise for health reasons alone. ${ }^{33}$ In fact, some patients who viewed physical activity as synonymous with health were less likely to be active if they noted any decline in their ability because this was viewed as evidence of deterioration in their health. While our finding does not contradict the EIM initiative per se, our research does extend previous thinking by highlighting the need for healthcare professionals to focus on the enjoyment, rather than solely the health benefits, of physical activity. This finding provides a tentative explanation as to why previous research has found that physical activity is not being promoted clinically, despite MDTs rating it as beneficial. ${ }^{27}$ Given the belief that physical 
Table 4 Approaches used to make activity normal

"We talk about the real importance of establishing an early routine in childhood of activity and exercise and from toddlers up to transition age we talk about developing good habits and trying to engage them with different sporting Quote 1 or different activities to give them the option and access to different types of activities" (James, paediatrician).

Quote 2 "We try to sort of make it part of their normal every day activity and explore avenues of families going for walks, bike rides, swimming or stuff like that" (Katie, physio).

Quote 3 "I think we need to talk more about health promotion and activity at school and potentially bring it into the school for everyone" (John, physio).

Quote 4 "The daily mile they do at school... making all the children go out and run a mile a day. I think that helps as well" (James, paediatrician).

Quote 5 "I think the general move across NHS England and having just the generally healthier population and making sports part of everybody's life means that they don't get picked out as someone who has to exercise because of their health they're doing it the same as everybody else is doing so making a much more normalised activity. So I think there are ways of society encouraging exercise as well" (Rachel, dietician).

Notes: All names provided are pseudonyms.

activity should be 'fun' rather than medicine, healthcare professionals may be reluctant to make physical activity a 'treatment' as this may diminish its appeal as a fun and enjoyable thing to do. Further research is needed to explore whether or not promotion of physical activity in clinics does reduce adolescents' enjoyment of physical activity, or indeed, change their perspective of physical activity from enjoyable to treatment.

In addition to identifying possible explanations for physical activity behaviour, the current study presents a number of strategies used to support and encourage adolescents to be active and enjoy the activity. While healthcare professionals may view some young people as lacking motivation for physical activity, previous qualitative research suggests that adolescents are often very active in managing their behaviours. ${ }^{34}{ }^{35}$ Indeed, Hughes $e t a l^{34}$ highlight the challenges faced by young people living with chronic conditions when attempting to live their lives, and how young people struggle to accommodate these interruptions. The role of moderating influences (eg, parents and healthcare professionals) in supporting the young person to develop behaviours to manage their illness alongside their everyday life is highlighted, alongside the need to develop interventions tailored to the needs and circumstances of individuals. Indeed, many of the approaches used by healthcare professionals centred around individualised recommendations, and approaches used to enhance intrinsic motivation and incorporate physical activity into everyday life. Healthcare professionals discussed how they would provide individuals with options and opportunities to try a range of activities in an attempt to identify one that may resonate with each individual and encourage friends and families to participate. However, there was also an acceptance that some adolescents do not enjoy physical activity, and do not have active friends and families. For these individuals, physical activity is not part of their everyday routine, and being active would just make them stand out from their peers, which adolescents are strongly motivated to avoid. ${ }^{36}$ In such cases, healthcare professionals reported attempts to make activity rewarding, either by offering rewards or treats at the end of activity sessions, or appealing to their competitive side with challenges and competitions (incentivising). Indeed, Segar and colleagues,${ }^{33}$ discussed the need to 'sell' physical activity to patients rather than focusing on clinicians' own goals, which are often irrelevant to their patients. The authors exemplify this using the pharmaceutical industry, demonstrating how they increase sales by promoting a behaviour for 'pleasure, happiness or quality time with family' (P.100). Healthcare professionals in the current study were strongly in agreement with this and recognised the need to promote physical activity in a way that resonates with their individual patients. By suggesting that 'enjoyment' is one of the most important factors in physical activity behaviour, our healthcare professionals highlight a feasible avenue for promoting, or selling, physical activity to their patients. Future research may now be concerned with exploring the effectiveness of such approaches to physical activity promotion in future interventions.

Social prescribing refers to attempts by healthcare professionals to link patients with non-clinical sources of support in the community ${ }^{37}$ and is advocated for people with chronic conditions. ${ }^{38}$ Within the current study, social prescribing (eg, suggesting young people attend park run) was used by healthcare professionals in an attempt to de-medicalise physical activity and make it a normal part of life. While further research into the effectiveness of social prescribing is needed, there is emerging evidence that it may lead to improvements in quality of life and emotional well-being. ${ }^{37}{ }^{39}$ Indeed, social prescribing may be particularly relevant for young people with $\mathrm{CF}$ who are aiming to exercise for benefits that reach beyond lung function. Further research exploring the potential of social prescribing for increasing physical activity among young people with $\mathrm{CF}$ is therefore required.

Theories of motivation highlight the need to target patients' intrinsic motivation ${ }^{37}$ in eliciting long-term, sustainable behavioural patterns. The theory articulates how motivation for a particular behaviour (ie, physical 
activity) is either intrinsic (self-determined) or extrinsic (driven by external or internal pressure such as guilt or coercion). The theory suggests that intrinsically motivated behaviours are more likely to be sustained in the long term. Findings from the current research are consistent with this theory, and suggest that healthcare professionals could focus on attempts to maximise intrinsic motivation for physical activity, given that attempts to enhance extrinsic motivation (eg, clinical pressure) are less enjoyable and less likely to be maintained over time. ${ }^{40}$

A large body of literature has identified BCTs that have the potential to influence self-determined motivation, ${ }^{32} 4142$ and healthcare professionals in the current study reported using many of these approaches, such as giving patients choice, encouraging rather than coercing, providing information and eliciting and acknowledging patients' perspectives. These approaches, consistent with both self-determination theory and motivational interviewing, highlight the need for healthcare professionals to identify exactly what it is that is important to patients, ${ }^{43}$ and to promote physical activity in a way that resonates with their goals. ${ }^{33} 40$ The finding that clinicians are using self-determination theory and motivational interviewing, although unknowingly, to change physical activity behaviour strongly supports the development of an intervention underpinned by self-determination theory. Moreover, incorporating elements of motivational interviewing may be effective in promoting physical activity for this audience. Existing research highlights the evidence for the effectiveness of interventions using motivational interviewing to promote physical activity among other clinical populations. ${ }^{24}$ Indeed, in the National Institute of Health and Care Excellence's guidance for 'making every contact count', they recommend that all healthcare professionals should be encouraged to deliver brief advice to motivate individuals for physical activity. It is therefore a potentially useful approach for motivating physical activity among people with CF.

Previously, attempts have been made to link the active mechanisms of the self-determination theory to BCTs. ${ }^{445}$ For example, Michie and colleagues ${ }^{44}$ highlight the links between the mechanisms of action (eg, 'social influences', 'motivation' and 'reinforcement') and BCT mentioned by healthcare professionals (eg, social rewards, incentives and social influences). Using a consensus approach, Teixeira and Haggar ${ }^{45}$ identified a number of BCTs relating to aspects of self-determination theory that correspond with practices identified by healthcare professionals in the current study, for example, 'facilitating discussion of clients view point'. This is entirely coherent with the current study, and suggests that, although healthcare professionals do not have any prior training in this area, they were able to identify similar approaches. Additional training in motivational interviewing, for example, may greatly enhance their practices.

\section{Strengths and limitations}

The main strength of this study is the use of qualitative methods to generate an in-depth account of the views of an understudied group: healthcare professionals working with adolescents with CF. The research team comprises researchers from multiple disciplines and with variable experience in qualitative research, and we acknowledge that our role in the research process will have influenced the direction of the research in terms of the questions we asked and, in our expectations, and interpretation of the data. While the perspective brought to the design and analysis is largely psychological and may have been influenced by the lead author's prior training in health psychology, every effort was made to enhance the trustworthiness of the present study. This included multiple coders (from multiple disciplines), the keeping of a reflective diary and respondent validation. ${ }^{31}$ Findings were also triangulated with the existing literature, and healthcare professionals were asked to validate our findings. ${ }^{31}$ Despite this, we acknowledge that researchers with different experiences and expectations may have reached different conclusions.

While the study used opportunistic sampling, 15 healthcare professionals from three disciplines across a large geographical area of the UK were incorporated. The use of opportunistic sampling may have resulted in a self-selected sample of individuals who are very interested in physical activity, and we were unable to collect data on the number of years of experience of our participants. Despite these limitations, all healthcare professionals were able to recognise and discuss approaches used to promote physical activity for adolescents with CF. Indeed, this population of interested healthcare professionals offer a unique perspective regarding the motivation of their patients and their attempts to promote physical activity, and may provide useful information for healthcare professionals who are less interested in physical activity.

The importance of considering the perspective of all stakeholders when developing interventions is recognised. ${ }^{25}$ Healthcare professionals have the advantage of being able to see a range of patients with wide variation in their physical activity levels and in a range of situations. This provides healthcare professionals with the unique opportunity to identify commonalities across patients in a way that patients themselves cannot. For example, while people with CF may be able to discuss factors that limit and restrict their physical activity behaviour, they would not be able to spot commonalities or patterns between individuals. Furthermore, healthcare professionals are able to discuss their experiences of using different strategies to increase motivation for physical activity. We suggest that future studies should be concerned with obtaining data from patients themselves, as this would complement the data we present in this study, and provide a comprehensive assessment of the situation. Integration of data obtained from multiple sources (eg, young people with $\mathrm{CF}$ ) is crucial for the 
development of effective interventions targeting physical activity for young people with $\mathrm{CF}^{25}$

To our knowledge, this is the first study exploring clinicians' perspectives relating to motivation and promotion strategies for physical activity among adolescents with CF, and has important implications for research and practice. First, converse to previous literature, ${ }^{27}$ our findings suggest that healthcare professionals could focus on the enjoyment element of physical activity, rather than its role in promoting health. Indeed, self-determination theory and motivational interviewing may offer a useful avenue for facilitating such autonomous motivation. In addition, eliciting social support, both family and peer, may further enhance enjoyment. The role of significant others recurred throughout the interviews, with healthcare professionals suggesting that friends and family can support the person with CF to be active by branding and marketing physical activity as both fun and normal. Providing such normalising experiences for youths may enhance their sense of identity and facilitate opportunities for peer relationships to develop. Indeed, it may be that there is a role for targeting patients' significant others in future interventions to promote physical activity. Future research should seek to explore the perspective of adolescents with CF, as well as the views of their friends and family, and subsequently assess the effectiveness of the collective strategies proposed.

\section{CONCLUSION}

Taken together, all healthcare professionals, irrespective of their role, felt that physical activity and exercise were crucial for adolescents with CF. Factors were identified that may influence patients' motivation to engage in physical activity and exercise; and in accord with research in healthy populations, individualised recommendations to enhance fun and enjoyment and integrate physical activity into everyday route were consistently cited for sustainable participation. In order to present a complete picture of the complexities of physical activity, future research should seek to explore perceptions of barriers and facilitators to physical activity from the perspective of young people with CF and their support teams. However, this work provides preliminary evidence for the suggestion that interventions developed around theories such as the self-determination theory may prove beneficial. This work provides a crucial first step in the intervention development process. ${ }^{25}$ Future work may now be concerned with exploring the views of stakeholders, including young people with $\mathrm{CF}$, and using data from multiple perspectives to inform the development of interventions to promote physical activity among young people with CF.

Acknowledgements We would like to thank the Cystic Fibrosis Trust for funding the research project, and for their support with recruitment of participants. We would also like to thank the volunteer practitioners for their valuable time in participating in this study. Members of YouthActivity Unlimited a Strategic Research Centre of the UK Cystic Fibrosis Trust are:Professor Craig Williams, Dr Alan Barker, Dr Sarah Denford, Dr Owen Tomlinson(University of Exeter, UK); Dr Patrick
Oades (Royal Devon and Exeter NHS Foundation Trust); Professor Eleanor Main, Dr Sarah Rand, Ms Helen Douglas(University College London, UK); Dr Mandy Byron (Great Ormond Street Hospitalfor Children, UK); Professor Anne Holland, Dr Narelle Cox, Dr Bev Eldridge, DrPaul O'Halloran (La Trobe University, Australia); Dr Kelly Mackintosh, DrMelita McNarry, Ms Mayara Silviera (Swansea University, UK); Dr JaneSchneiderman, Dr Greg Wells, Ms Jessica Caterini (Hospital for Sick Children,Canada).

Contributors The study was designed by SD with input from CAW, AB, KAM and MAM. Data were collected and analysed by SD with considerable input from KAM and MAM. The manuscript was prepared by SD with considerable input from CAW, $A B, K A M$ and MAM. All authors approved the final manuscript.

Funding This work was funded by the Cystic Fibrosis Trust Strategic Research Centre grant number 008.

Competing interests None declared.

Patient consent for publication Not required.

Ethics approval All procedures performed in studies involving human participants were in accordance with the ethical standards of the institutional and/or national research committee and with the 1964 Helsinki declaration and its later amendments or comparable ethical standards. Ethical approval was obtained from the University of Exeter institutional Ethics Committee (161207/A/03).

Provenance and peer review Not commissioned; externally peer reviewed.

Data sharing statement № additional data are available.

Open access This is an open access article distributed in accordance with the Creative Commons Attribution Non Commercial (CC BY-NC 4.0) license, which permits others to distribute, remix, adapt, build upon this work non-commercially, and license their derivative works on different terms, provided the original work is properly cited, appropriate credit is given, any changes made indicated, and the use is non-commercial. See: http://creativecommons.org/licenses/by-nc/4.0/.

\section{REFERENCES}

1. Cystic Fibrosis Trust. UK Cystic fibrosis registry 2016 Annual Data Report. 2017a.

2. Barr HL, Britton J, Smyth AR, et al. Association between socioeconomic status, sex, and age at death from cystic fibrosis in England and Wales (1959 to 2008): cross sectional study. BMJ 2011;343:d4662.

3. Cystic Fibrosis Trust. About cystic fibrosis. 2013. Available at: http://www.cysticfibrosis.org.uk/about-cf/publications/consensusdocuments.aspx

4. Sawicki GS, Sellers DE, Robinson WM. High treatment burden in adults with cystic fibrosis: challenges to disease self-management. $J$ Cyst Fibros 2009;8:91-6.

5. Savage E, Beirne PV, Ni Chroinin M, et al. Self-management education for cystic fibrosis (Cochrane Review). Cochrane Databse of Systematic Reviews (9. Art. No: CD007641) 2014.

6. Cystic Fibrosis Trust. Standards of care and good clinical practice for the physiotherapy management of cystic fibrosis. 3rd edn: The Cystic Fibrosis Trust, 2017b.

7. Bradley J, Moran F. Physical training for Cystic Fibrosis. Cochrane Databse of Systematic Reviews 2008;23:CD002768.

8. Stanghelle JK, Koss JO, Bjørtuft O, et al. Marathon with cystic fibrosis and bilateral lung transplant. Scand J Med Sci Sports 2000;10:42-6.

9. Prasad SA, Cerny FJ. Factors that influence adherence to exercise and their effectiveness: application to cystic fibrosis. Pediatr Pulmonol 2002;34:66-72.

10. Street R, Mercer J, Mills-Bennett R, et al. Experiences of physical activity: A phenomenological study of individuals with cystic fibrosis. $J$ Health Psychol 2016;21:261-70.

11. Fereday J, MacDougall C, Spizzo M, et al. "There's nothing I can't do - I just put my mind to anything and I can do it": a qualitative analysis of how children with chronic disease and their parents account for and manage physical activity. BMC Pediatr 2009;9:1-16.

12. Selvadurai HC, Blimkie CJ, Cooper PJ, et al. Gender differences in habitual activity in children with cystic fibrosis. Arch Dis Child 2004;89:928-33.

13. Vancampfort D, Stubbs B, Koyanagi A. Physical chronic conditions, multimorbidity and sedentary behavior amongst middle-aged and older adults in six low- and middle-income countries. Int J Behav Nutr Phys Act 2017;14:147. 
14. Mackintosh KA, Ridgers ND, Evans RE, et al. Physical activity and sedentary time patterns in children and adolescents with cystic fibrosis and age- and sex-matched healthy controls. J Phys Act Health 2018;15:82-8.

15. Hebestreit H, Schmid K, Kieser S, et al. Quality of life is associated with physical activity and fitness in cystic fibrosis. BMC Pulm Med 2014;14:26.

16. Pianosi P, Leblanc J, Almudevar A. Peak oxygen uptake and mortality in children with cystic fibrosis. Thorax 2005;60:50-4.

17. Schneiderman JE, Wilkes DL, Atenafu EG, et al. Longitudinal relationship between physical activity and lung health in patients with cystic fibrosis. Eur Respir J 2014;43:817-23.

18. Deci EL, Ryan RM. Intrinsic motivation and self-determination in human behavior. New York: Plenum, 1985.

19. Lubans DR, Lonsdale $\mathrm{C}$, Cohen $\mathrm{K}$, et al. Framework for the design and delivery of organized physical activity sessions for children and adolescents: rationale and description of the 'SAAFE' teaching principles. Int J Behav Nutr Phys Act 2017;14:24.

20. Sweet SN, Fortier MS, Strachan SM, et al. Testing a longitudinal integrated self-efficacy and self-determination theory model for physical activity post-cardiac rehabilitation. Health Psychol Res 2014;2:1008.

21. Miller WR, Rose GS. Toward a theory of motivational interviewing. Am Psychol 2009;64:527-37.

22. Deci EL, Ryan RM. Self-determination theory in health care and its relations to motivational interviewing: a few comments. Int J Behav Nutr Phys Act 2012;9:24.

23. Patrick H, Williams GC. Self-determination theory: its application to health behavior and complementarity with motivational interviewing. Int J Behav Nutr Phys Act 2012;9:18.

24. Britt E, Hudson SM, Blampied NM. Motivational interviewing in health settings: a review. Patient Educ Couns 2004;53:147-55.

25. Craig P, Dieppe P, Macintyre S, et al. Developing and evaluating complex interventions: the new Medical Research Council guidance. Int J Nurs Stud 2013;50:587-92.

26. Braun V, Clarke V. What can "thematic analysis" offer health and wellbeing researchers? International Journal of Qualitative Studies on Health and Well-Being. 9.Artn 2014;26152.

27. Stevens D, Oades PJ, Armstrong N, et al. A survey of exercise testing and training in UK cystic fibrosis clinics. J Cyst Fibros 2010;9:302-6.

28. Riekert KA, Eakin MN, Bilderback A, et al. Opportunities for cystic fibrosis care teams to support treatment adherence. J Cyst Fibros 2015;14:142-8.
29. Miles MB, Huberman AM. Qualitative Data Analysis: An expanded source book. 2nd edn. Thousand Oaks: Sage Publications, 1994.

30. Ritchie J, Spencer L. Qualitative data analysis for applied policy research. The qualitative researcher's companion 2002;573:305-29.

31. Yardley L. Dilemmas in qualitative health research. Psychol Health 2000;15:215-28.

32. Lobelo F, Stoutenberg M, Hutber A. The Exercise is Medicine Global Health Initiative: a 2014 update. Br J Sports Med 2014;48:1627-33.

33. Segar ML, Guérin E, Phillips E, et al. From a vital sign to vitality: Selling exercise so patients want to buy It. Curr Sports Med Rep 2016;15:97-102.

34. Hughes M, Savage E, Andrews T. Accommodating interruptions: A grounded theory of young people with asthma. J Clin Nurs 2018;27:212-22.

35. Winn CON, Mackintosh KA, Eddolls WTB, et al. Perceptions of asthma and exercise in adolescents with and without asthma. $J$ Asthma 2018;55.

36. Segal TY. Adolescence: what the cystic fibrosis team needs to know. $J$ S Soc Med 2008;101 Suppl 1(Suppl 1):15-27.

37. Segar ML, Richardson CR. Prescribing pleasure and meaning: cultivating walking motivation and maintenance. Am J Prev Med 2014;47:838-41.

38. Bickerdike L, Booth A, Wilson PM, et al. Social prescribing: less rhetoric and more reality. A systematic review of the evidence. BMJ Open 2017;7:e013384

39. Department of Health. White paper. Our health, our care, our say: a new direction for community services. Crown copyright. 2006.

40. The Kingsfund. What is social prescribing. 2017 https://www. kingsfund.org.uk/publications/social-prescribing.

41. Deci EL, Eghrari H, Patrick BC, et al. Facilitating internalization: the self-determination theory perspective. J Pers 1994;62:119-42.

42. Silva MN, Vieira PN, Coutinho SR, et al. Using self-determination theory to promote physical activity and weight control: a randomized controlled trial in women. J Behav Med 2010;33:110-22.

43. Denford S, Frost J, Dieppe P, et al. Doctors' understanding of individualisation of drug treatments: a qualitative interview study. BMJ Open 2013:3:e002706.

44. Michie S, Johnston M, Rothman AJ, et al. Developing methodology for designing and evaluating theory-based complex interventions: an ontology for linking behaviour change techniques to theory, funded by the UK Medical Research Council, 2014.

45. Teixeira P, Hagger M. Motivation and behaviour change techniques based on self-determination theory: a consensus approach. Bulletin of the European Health Psychology Society 2016;18. 